\title{
Atlantic salmon raised with diets low in long-chain polyunsaturated $n-3$ fatty acids in freshwater have a Mycoplasma-dominated gut microbiota at sea
}

\author{
Yang Jin ${ }^{1,3}$, Inga Leena Angell ${ }^{2}$, Simen Rød Sandve ${ }^{3}$, Lars Gustav Snipen², \\ Yngvar Olsen ${ }^{1}$, Knut Rudi ${ }^{2, *}$ \\ ${ }^{1}$ Department of Biology, Norwegian University of Science and Technology, 7491 Trondheim, Norway \\ ${ }^{2}$ Faculty of Chemistry, Biotechnology and Food Science, University of Life Sciences, 1430 Ås, Norway \\ ${ }^{3}$ Centre for Integrative Genetics, Department for Animal and Aquacultural Sciences, Faculty of Biosciences, \\ University of Life Sciences, 1430 Ås, Norway
}

\begin{abstract}
Factors affecting the establishment of the gut microbiota in animals living in marine environments remain largely unknown. In terrestrial animals, however, it is well established that the juvenile environment has a major impact on the gut microbiota later in life. Atlantic salmon Salmo salar is an anadromous fish important in aquaculture with a juvenile freshwater stage and an adult seawater stage. For wild salmon, there are major dietary changes with respect to availability of long-chain polyunsaturated n-3 fatty acids (LC-n-3 PUFA) with lower abundance in freshwater systems. The aim of our work was therefore to determine the effect of a juvenile freshwater diet with high LC-n-3 PUFA, as compared to a diet low in LC-n-3 PUFA (designed to increase the endogenous LC-n-3 PUFA production), on the transition to a seawater gut microbiota for Atlantic salmon. We found a juvenile freshwater microbiota high in Firmicutes for fish raised with low LC-n-3 PUFA, while the microbiota for fish given high LC-n-3 PUFA feed was high in Proteobacteria. One hundred days after transfer to a common sea cage, fish that were given low LC-n-3 PUFA diets in freshwater showed significantly higher ( $\mathrm{p}=0.02$, Kruskal-Wallis) Mycoplasma content ( $90 \pm 7 \%$; mean \pm SD) compared to fish raised on a high LC-n-3 PUFA diet in freshwater ( $25 \pm 31 \%$ Mycoplasma). Shotgun metagenome sequencing from fish raised with a low LC-n-3 PUFA diet identified a salmon-associated Mycoplasma in sea, being distinct from currently known Mycoplasma. The genome sequence information indicated a mutualistic lifestyle of this bacterium. Mycoplasma has also previously been identified as dominant (>70\%) in sea-living adult Atlantic salmon. Taken together, our results suggest that the juvenile freshwater diet influences the establishment of the gut microbiota in marine Atlantic salmon.
\end{abstract}

KEY WORDS: LC-n-3 PUFA · Marine stage $\cdot$ Freshwater stage $\cdot$ qPCR $\cdot$ 16S rRNA-metagenome

\section{INTRODUCTION}

Our knowledge about host-microbe interactions in fish across environmental transitions remains limited. Anadromous fish that migrate between freshand seawater, such as Atlantic salmon Salmo salar, experience a dramatic shift in both biotic and abiotic environments, requiring complete remodeling of osmoregulation from a hypotonic freshwater to a

\footnotetext{
*Corresponding author: knut.rudi@nmbu.no
}

hypertonic seawater environment (Lien et al. 2016). In addition to the change in osmolarity, there are major differences in fresh- and seawater in the availability of nutrient sources and thus dietary composition, such as the availability of essential long-chain polyunsaturated n-3 fatty acids (LC-n-3 PUFA), including eicosapentaenoic acid (EPA, 20:5n-3) and docosahexaenoic acid (DHA, 22:6n-3) (Leaver et al. 2008).

(C) The authors 2019. Open Access under Creative Commons by Attribution Licence. Use, distribution and reproduction are unrestricted. Authors and original publication must be credited. 
Particularly important in the salmon life cycle is the first migration from freshwater to the sea. Recent evidence indicates that this process is associated with both a major increase in abundance of the microbiota, with about 100-fold increase in bacterial load (Rudi et al. 2018), and a change in the microbiota composition, with an increase in Firmicutes and Tenericutes (Llewellyn et al. 2016, Dehler et al. 2017, Rudi et al. 2018). For terrestrial vertebrates, it is well documented that the interplay between environment, diet and gut microbiota at the juvenile stage can have a major impact on the microbiota- and immunological development at a later stage in life (Rodriguez et al. 2015). Knowledge is lacking on how juvenile freshwater management and diets (Buttigieg \& Ramette 2014) can have a long-term effect on the seawater-associated gut microbiota for salmon (Holben et al. 2002, Llewellyn et al. 2016). Such information, however, could be important for future farming strategies to improve fish health (Limborg et al. 2018).

Farmed salmon are given a diet high in LC-n-3 PUFA at the freshwater stage to increase the growth rate (Gillard et al. 2018). However, a high LC-n-3 PUFA diet in freshwater deviates from the natural diet (Bell et al. 1994). The aim of our work was therefore to compare a high LC-n-3 PUFA diet (the current commercial diet) with a low LC-n-3 PUFA diet (designed to increase the intrinsic LC-n-3 PUFA production) at the freshwater stage with respect to the transition from a juvenile- to an adult-like gut microbiota for Atlantic salmon.

\section{MATERIALS AND METHODS}

\subsection{Experimental design}

The study was carried out within the Norwegian Animal Welfare Act guidelines, in accordance with EU regulations (EC Directive 86/609/EEC) and was approved by the Norwegian Animal Research Authority (NARA). Atlantic salmon eggs were hatched and cultivated at the AquaGen Breeding Centre (Kyrksæterøra, Norway). The fish were divided into 2 experimental groups in the freshwater phase. One group was maintained as in normal commercial production and given a standard marineoil-based diet with high LC-n-3 PUFA such as EPA and DHA, while the other group was maintained in conditions more similar to that of a natural freshwater diet and given a low LC-n-3 PUFA diet using alternative n-3 PUFA such as alpha-linolenic acid
(ALA, 18:3n-3) to replace marine LC-n-3 PUFA. Both experimental groups were maintained with the same water source in freshwater. There were in total $\sim 3000$ fish in each experimental group. The fishes were first maintained in closed tanks measuring $0.5 \mathrm{~m}^{3}$, with transfer to $5 \mathrm{~m}^{3}$ after the first feeding. In sea, the fish were transferred to a commercial cage, containing about 60000 fish. The fish were passive integrated transponder (PIT)-tagged prior to sea transfer. All fish were moved to a common cage when transferred to seawater and then given the high LC-n-3 PUFA diet with a fatty acid composition comparable to that of the natural marine diet. The composition of the 2 diets and their fatty acids are summarized in Table S1 in the Supplement at www.int-res.com/articles/suppl/m011 p031_supp.pdf, while data on host responses are published elsewhere (Jin et al. 2018).

To better prepare Atlantic salmon for seawater transfer, the fish were exposed to a period of shorter days (12 h photoperiod, Meyer et al. 2008) and cooler water temperature $\left(8^{\circ} \mathrm{C}\right)$ to simulate winter. Fish were kept under winter conditions for $5 \mathrm{wk}$. Due to differences in growth rates, the 2 groups of fish were shifted to winter conditions at 113 (high LC-n-3 PUFA) and 120 (low LC-n-3 PUFA) d after the first feeding, respectively. The fish were then transferred to spring conditions $\left(24 \mathrm{~h}\right.$ photoperiod and $12^{\circ} \mathrm{C}$ water temperature) for $6 \mathrm{wk}$ before being transferred to a common sea cage at 205 (high LC-n-3 PUFA) and 211 (low LC-n-3 PUFA) d after the first feeding, respectively.

\subsection{Sampling and DNA isolation}

Fish were sampled at Day 114 (n = 8 for low LCPUFA and $n=10$ for low LC-PUFA), Day 150 and Day 157 (high and low LC-PUFA group, respectively, during winter, $\mathrm{n}=15$ for each group), Day 204 ( $\mathrm{n}=15$ for low LC-PUFA group and $\mathrm{n}=14$ for low LC-PUFA) and Day 320 (sea cage, $\mathrm{n}=5$ for each group) after the first feeding. An overview of the experimental setup is given in Fig. 1.

The fish were euthanized by $1 \mathrm{~g} \mathrm{l}^{-1} \mathrm{MS}-222$ (FINQUEL, Argent Chemical Labs) buffered with the same amount of sodium bicarbonate $\left(1 \mathrm{~g} \mathrm{l}^{-1}\right)$. The fish were dissected with a sterile blade and the gut content was gently squeezed into a $2 \mathrm{ml}$ microtube (Sarstedt) containing $0.5 \mathrm{ml}$ stool transport and recovery buffer (Roche) and $200 \mathrm{mg}$ glass beads of $100 \mu \mathrm{m}$ size. The tubes were kept at $4^{\circ} \mathrm{C}$ for $3-4 \mathrm{~h}$ before they were transferred to $-80^{\circ} \mathrm{C}$ for long-term storage. DNA 


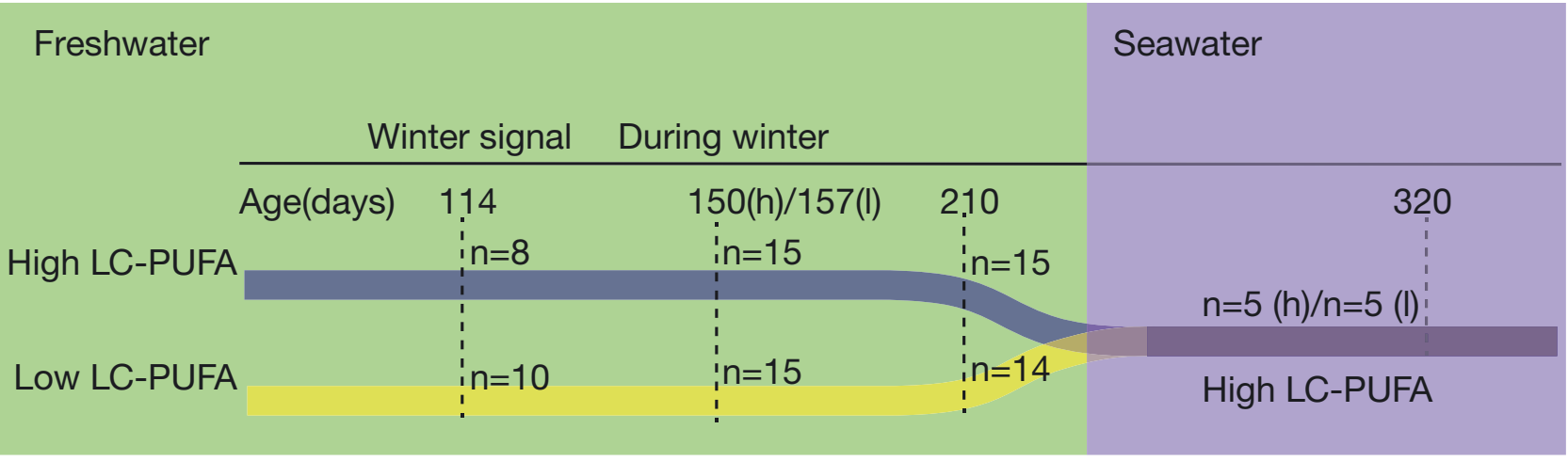

Fig. 1. Experimental design. Two tanks were used at the freshwater stage, while all fish (Salmo salar) were co-located in the same tank in seawater. Vertical dashed lines indicate sampling days. For each sampling point, the number of fish analyzed is indicated. LC-PUFA = long-chain polyunsaturated fatty acids, $\mathrm{h}=\operatorname{diet}$ high in LC-PUFA, $1=$ diet low in LC-PUFA

was extracted using magnetic separation with a 'mag midi' kit (LGC Genomics) according to the manufacturer's instructions in an automated KingFisher Flex purification system (Thermo Fisher Scientific). Quantification was done using a Qubit dsDNA HS (highsensitivity) assay kit (Thermo Fisher Scientific).

\subsection{Metagenome sequencing and analysis}

To determine taxonomic composition, library construction and sequencing of resulting amplicons of the V3-V4 region of the 16S rRNA was done as previously described (Rudi et al. 2018) for all the samples $(\mathrm{n}=87)$. Raw data was extracted for barcodes, forward and reverse reads were joined, and libraries split and merged into 1 large sequencing file. Further processing was done using usearch 8.0 (Edgar 2010). Low-quality reads (minimum length set to $350 \mathrm{bp}$, maximum expected error: 1.0) were removed before the sequences were dereplicated (using the command -derep_fullength) and sorted by abundance (using the command -sortbysize). Singletons were removed (default setting of usearch $=$ minimum abundance of 2). Clustering of the sequences was done using the Uparse-OTU algorithm implemented in usearch with an identity threshold of $97 \%$ (cluster_otus command). A chimera check using uchime with the greengenes database v.13_8 (DeSantis et al. 2006) was done in addition (using the command -uchime_ref). Sequences were assigned taxonomy using QIIME v.1.9.1 (Caporaso et al. 2010) (assign_ taxonomy.py script) using uclust and the greengenes database v.13_8. A uniform sequence depth of 10000 sequences per sample was used for further analysis.

To fully characterize the microbiota, 4 samples from Day 320 (fish from sea cage) were chosen for whole-genome shotgun sequencing. A sequencing library was constructed using a Nextera XT kit (Illumina) following the manufacturer's recommendations. Sequencing was performed on a MiSeq platform (Illumina) using a 600-cycle MiSeq v3 reagent kit.

For the shotgun analyses, each data set was processed separately, unless stated otherwise. First, we used Trimmomatic (Bolger et al. 2014) to qualityfilter and trim raw reads. Next, we used Bowtie2 (Langmead \& Salzberg 2012) to map all read-pairs to an index built from the salmon genome and transcriptome, to discard host contaminants. The remaining reads were assembled using metaspades (Nurk et al. 2013). Next, we again used Bowtie2 to map all reads back to the assembled contigs, and used the JGI script from MetaBat (Kang et al. 2015) to compute coverages and coverage variances for all contigs. Then MetaBat was used for binning of the contigs.

The contigs were then uploaded to MG-RAST (Keegan et al. 2016) for annotation. The gene annotations from the databases GenBank, RefSeq, IMG, SEED, TrEMBL, PATRIC and KEGG were retrieved for each data set from the MG-RAST server. Based on the taxon assignment for each gene annotation from each database, we computed a majority-vote genus classification for each contig. Some of the longer contigs had comparatively few annotated genes, resulting in a somewhat uncertain taxon assignment by this approach. Thus, in addition to the MG-RAST results, all contigs were also classified by Kraken2 (Wood \& Salzberg 2014). A $k$-mer-based method like Kraken2 is presumed to perform well on longer contigs, and complement the results from MG-RAST in this sense.

The final classification of the contigs were obtained as follows: 
(1) All contigs in the same bin were given the majority classification unless both Kraken2 and MGRAST results suggested otherwise.

(2) An unbinned contig was added to a bin if both Kraken2 and MG-RAST results suggested the same taxon as the bin, and the GC content was in the same range.

In an effort to quantify the similarity to already sequenced genomes, we first used the Prokka pipeline (Seemann 2014) to predict and annotate genes from the classified contigs. Next, we downloaded all complete genomes of the genera Mycoplasma and Photobacterium from NCBI/Genome. The exact same Prokka pipeline was used on these genomes. All genes listed as 'hypothetical protein' were discarded, and a Jaccard distance was computed between all pairs of genomes. This reflects the similarity in annotated genes, i.e. if 2 genomes have the exact same gene annotations, the Jaccard distance between them is 0 . A standard multidimensional scaling was used on the Jaccard distance matrix to visualize how the genomes com-pare.

\subsection{Quantitative PCR}

To quantify bacterial load, both eukaryote DNA (V4 region of the 18S rRNA gene) and prokaryote DNA (V3-V4 region the of 16S rRNA gene) were quantified by performing quantitative PCR (qPCR) using the HotFirePol Evagreen qPCR supermix (Solis BioDyne) and the primer pairs PRK341 (5'-CCT ACG GGR BGC ASC AG-3') and PRK806R (5'-GGA CTA CYV GGG TAT CTA AT-3') (Yu et al. 2005), and 3NDF (5'-GGC AAG TCT GGT GCC AG-3') and V4EukR2 (5'-ACG GTA TCT RAT CRT CTT CG-3') (Bråte et al. 2010), as described previously (Angell et al. 2017).

\subsection{Statistical analyses}

The statistical analyses were done using either Minitab v.18, or Matlab 2016b (MathWorks) with the PLS toolbox plugin (Eigenvector).

\section{RESULTS}

\subsection{Overall microbiota composition determined by $16 \mathrm{~S}$ rRNA gene analyses}

We obtained a total of 2071018 sequences from 87 distal intestinal samples (Fig. 2). The samples were rarified at 10000 sequences, with 84 samples satisfying the rarefaction criteria (Fig. 2A). All the rarified samples had Good's coverage > $99 \%$. We detected in total 750 OTUs, with the highest and lowest OTU richness for the low LC-PUFA group at 157 and 322 $\mathrm{d}$, with respective medians of 149 and $24.5(\mathrm{p}=0.003$, Kruskal-Wallis test). In freshwater, we detected major differences in the composition of the microbiota between the high and low LC-PUFA groups $(\mathrm{R}=0.27$,
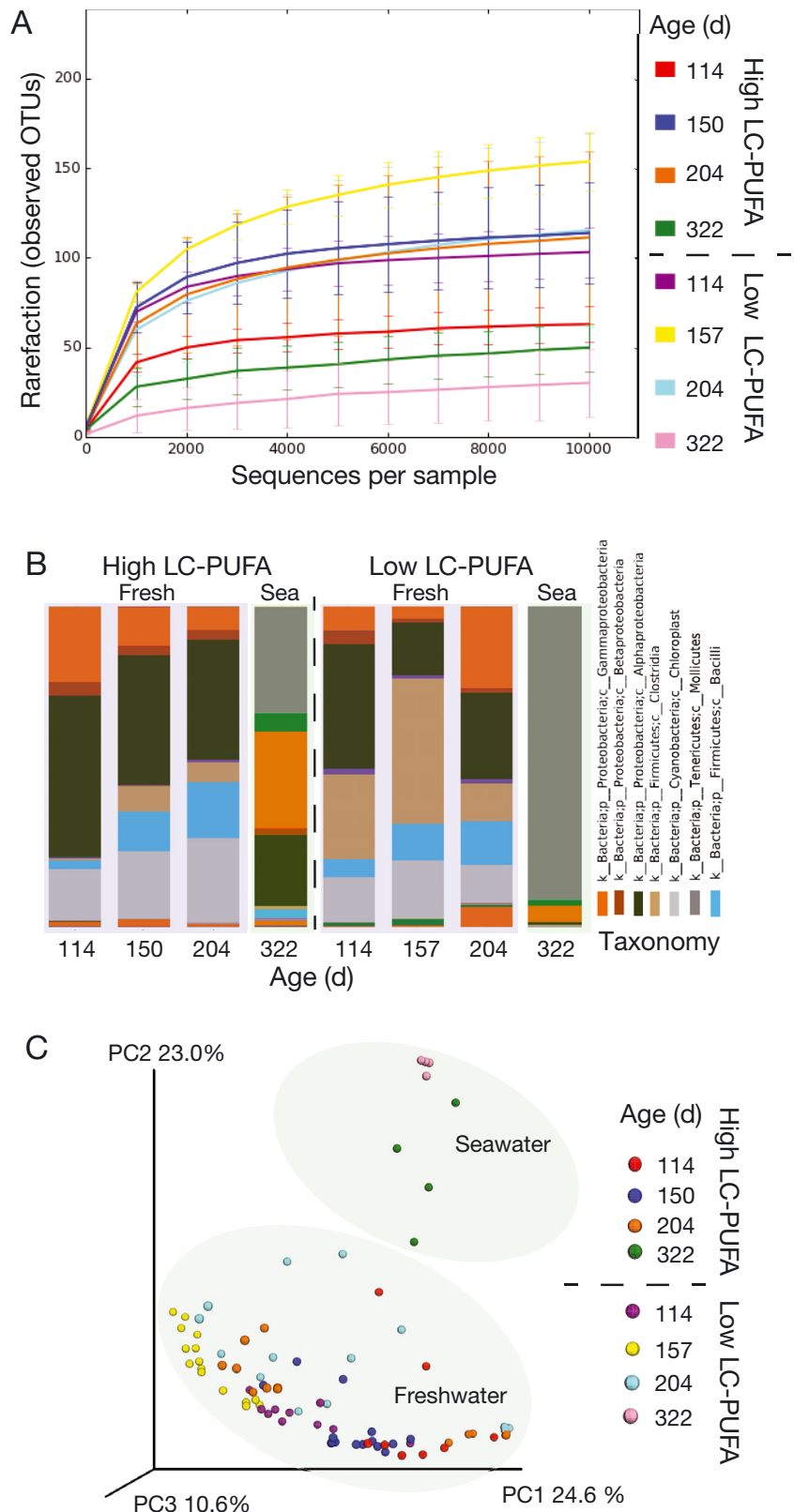

Fig. 2. Overall microbiota composition. (A) Rarefaction analyses, (B) taxonomic composition, and (C) beta diversity, as visualized by Bray-Curtis principal coordinate analysis. Age and diet indicated by a color code. Graphics were generated using the QIIME pipeline (Caporaso et al. 2010).

LC-PUFA = long-chain polyunsaturated fatty acids 
$\mathrm{p}=0.001$, ANOSIM $_{i}$ Fig. 2C). Firmicutes showed the largest difference, with a major overrepresentation for low LC-PUFA, while Proteobacteria was overrepresented for high LC-PUFA (Fig. 2B). We found a complete remodeling of the microbiota after $100 \mathrm{~d}$

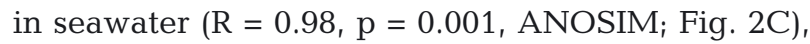
with a dominance of Mycoplasma for high LC-PUFA and Photobacterium for low LC-PUFA (Fig. 2B).

\subsection{Fish growth and microbiota development}

The high LC-PUFA fish had a significantly higher growth rate than the low LC-PUFA fish, while the difference was evened out in the seawater stage (Fig. 3A, Table S2 in the Supplement). There was also a major increase in bacterial load (Fig. 3B) and a decrease in alpha diversity in seawater compared to freshwater, with no differences in bacterial load or alpha diversity between high and low LC-PUFA fish (Fig. 3C,D).
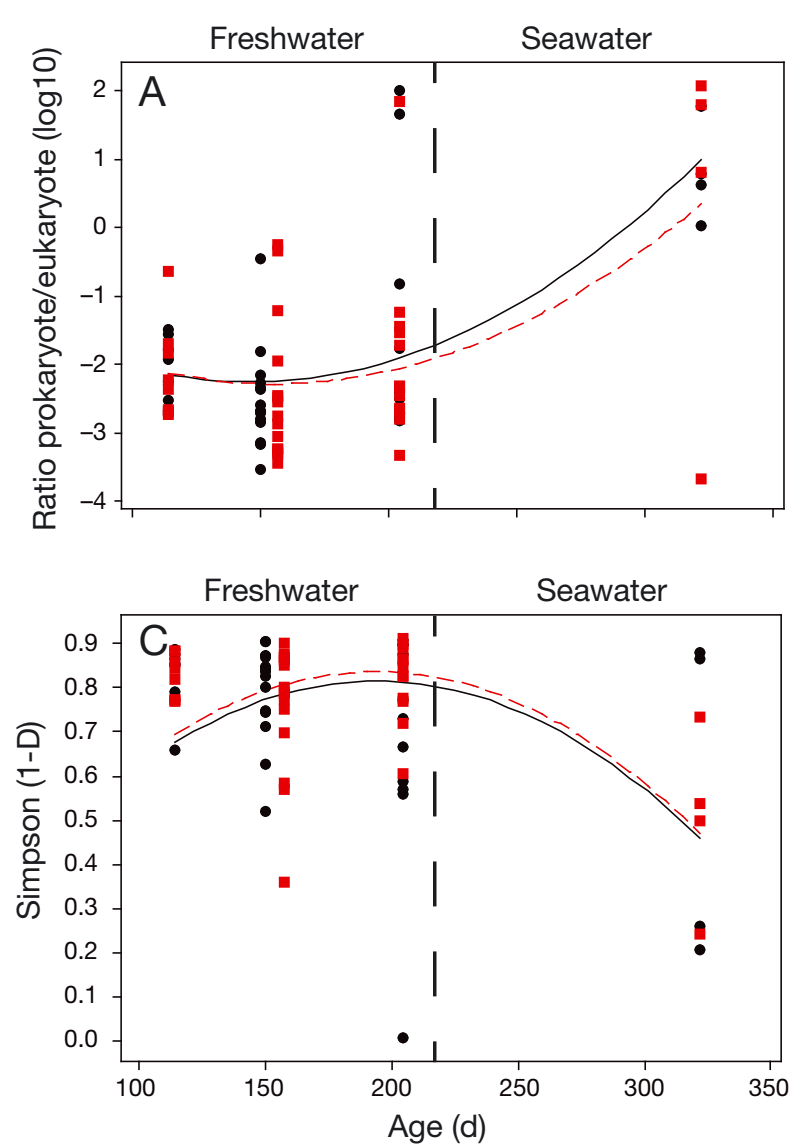

We identified the OTUs 2, 8, 27 and 45 sharing $>97 \%$ identity to bacteria previously identified as core bacteria (Rudi et al. 2018). The relative abundance of these OTUs in each sample was highly correlated (for all pairwise comparisons: Spearman's rho $>0.79$, $\mathrm{p}<0.0001$ ). Because of this correlation within each sample, we summed these abundances in each sample and analyzed the variation in total abundance of core associated OTUs, referred to as the 'core index'. This index showed a major peak at $157 \mathrm{~d}$ for the low LCPUFA conditions, while the levels were relatively low throughout the experiment for the high LC-PUFA conditions (Fig. 4A). Mycoplasma contrasted the development of the core microbiota, with a major expansion in the sea for the fish originating from the low LC-PUFA conditions, and a less pronounced expansion for the fish from the high LC-PUFA conditions (Fig. 4B). For salmon in sea, we found $90 \pm 7 \%$ (mean \pm SD) $M y$ coplama for the low LC-PUFA group, while the high LC-PUFA showed $25 \pm 31 \%$ Mycoplama, representing a significant difference $(\mathrm{p}=0.02$, Kruskal-Wallis).
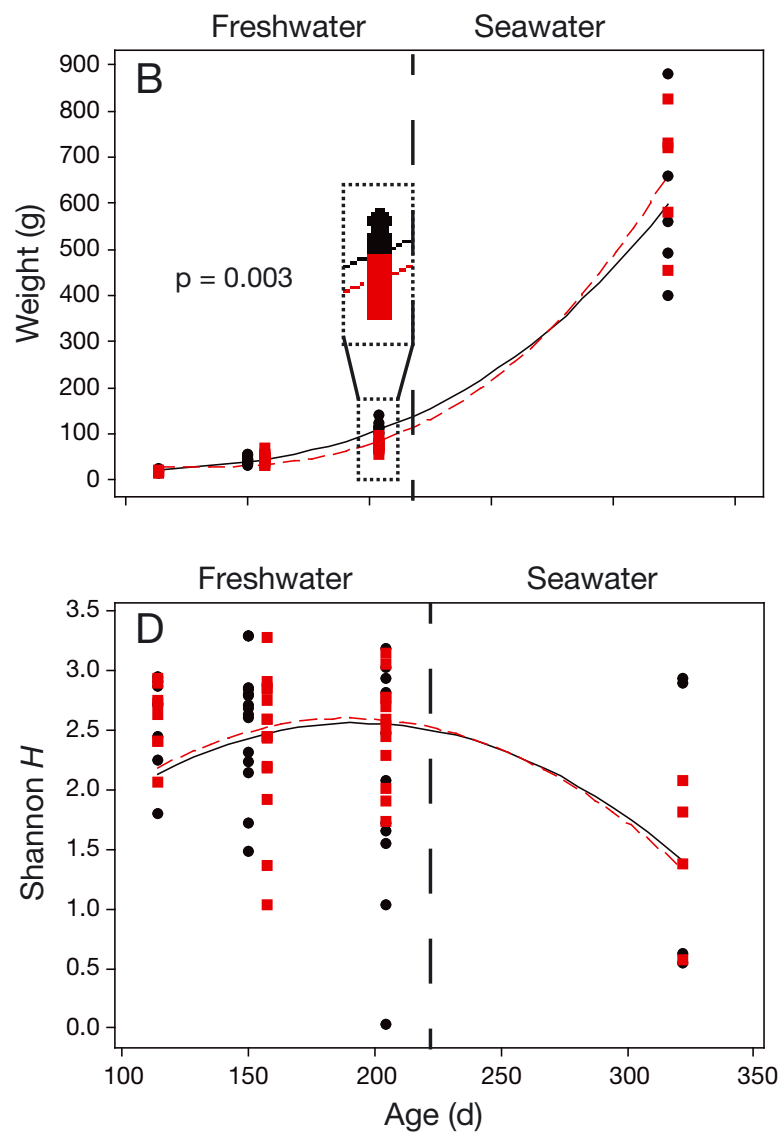

Fig. 3. Age-related development of (A) bacterial load, (B) weight, and (C,D) alpha diversity (C: Simpson index, D: Shannon index), for high long-chain polyunsaturated fatty acids (LC-PUFA) (black circle) and low LC-PUFA (red square) freshwater conditions. For (A), bacterial load was determined by the ratio of eukaryote 18S rRNA and prokaryote 16S rRNA gene copies, since this ratio is not affected by the total amount of material analyzed. In (B), the difference in weight at $204 \mathrm{~d}$ (dashed outlines) is significant (Kruskal-Wallis test) 

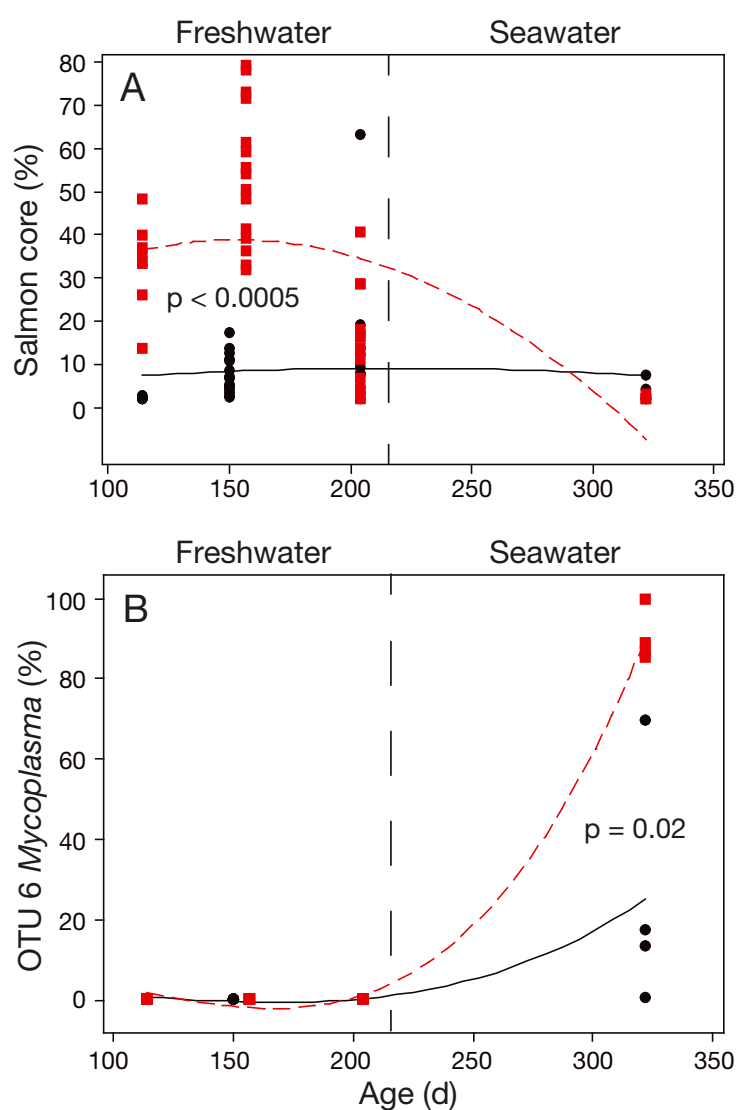

Fig. 4. Age-related development of (A) core microbiota and (B) Mycoplasma for high long-chain polyunsaturated fatty acids (LC-PUFA) (black circle) and low LC-PUFA (red square) freshwater conditions. Significance determined by Kruskal-Wallis test

\subsection{Shotgun metagenome sequencing of seawater gut microbiota}

Three of the 4 metagenome data sets produced assembled contigs of a meaningful quality (lengths/ coverages), as summarized in Table S3 in the Supplement. For the 2 sets with most reads, contigs were binned into 3 distinct groups by MetaBat, while for the third and smaller set, only 1 distinct bin was detected in addition to all unbinned contigs. The taxon classifications, by either Kraken2 or by majority vote from the MG-RAST annotations, corresponded well by the binning. One bin from each data set was classified as Mycoplasma. The other 2 bins, in the 2 larger data sets, were both classified as Photobacterium.

In Fig. 5, we show the binned contigs for sample Kyrk.E3 (from the low LC-PUFA group). The blue dots correspond to 2 bins, both classified as Photobacterium. We also BLASTed the centroid reads from the 16S data to the contigs, and OTU6 (Mycoplasma) produced $>99 \%$ matches against some contigs classified as Mycoplasma, and OTU102 and OTU379 (Photobacterium) produced $>98 \%$ and $>97 \%$ matches against contigs classified as Photobacterium.

The annotations of the Mycoplasma contigs resulted in around 600-700 coding genes (discarding 'hypothetical proteins') in each, which is in the same range as we see in complete genomes of this genus. In Fig. 6A, we have plotted a principal coordinate ordination of the Jaccard distances between the annotations of the Mycoplasma genomes. First, we notice the 3 genomes in our metagenome samples (blue dots) are very similar to each other, and most probably this is the same organism in all 3 fish. We also notice that, given the variation between complete Mycoplasmas, this organism is quite similar, but not identical, to the cluster of genomes near $M$. penetrans and near $M$. yeatsii. Venn diagram analyses revealed that the salmon Mycoplasma contained the lowest number of unique genes compared to the closely related groups (Fig. 6B). Annotations for the unique genes indicate that there is an overrepresentation of carbohydrate uptake genes (PTS) for both the salmon Mycoplasma (5.8\%, 4 out of 64$)$ and the $M$. yeatsii group $(5.0 \%, 12$ out of 242$)$, as compared to the M. penetrans group $(1.3 \%, 4$ out of 307$)$. The level of peptidases, however, was lowest in salmon Mycoplasma (1.5\%, 1 out of 68$)$, as compared to the M. penetrans $(4.9 \%, 15$ out of 307$)$ and the $M$. yeatsii group (3.7\%, 9 out of 242$)$. A full list of unique genes for the different groups is provided in Table S4 in the Supplement.

A similar plot was also constructed for Photobacterium, but is not shown here. There are currently only 3 complete Photobacterium genomes to compare against $(P$. damselae, $P$. gaetbulicola, $P$. profundum). The contigs from sample Kyrk.C5 (from the high LC-PUFA group) resulted in $\sim 3100$ annotated genes, which is similar to P. damselae. This was also the nearest neighbor of the contigs from this sample. The contigs from sample Kyrk.E3 only produced $\sim 2000$ annotated genes, and was therefore not very close to any complete genome.

\section{DISCUSSION}

The low LC-PUFA juveniles showed the largest resemblance to wild salmon in the marine stage, with a major dominance of Mycoplasma (Llewellyn et al. 2016). In our experiment, we found $90 \pm 7 \%$ Mycoplama for the low LC-PUFA group, while the high LC-PUFA showed $25 \pm 31 \%$ Mycoplama. In 
Binned contigs from fish Kyrk.E3 (low LC-PUFA)

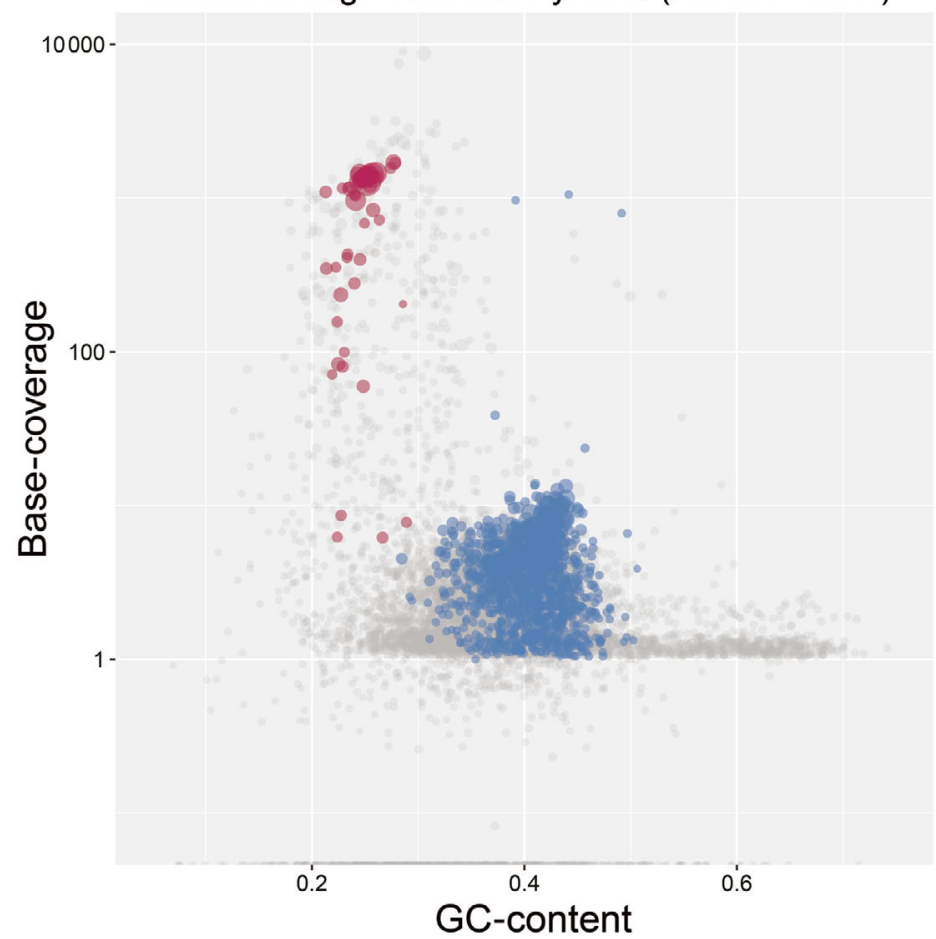

Fig. 5. Contig binning. Each dot corresponds to an assembled contig, positioned according to its GC content and base coverage. Colored dots correspond to contigs classified as Mycoplasma (red) or Photobacterium (blue). Note that the blue dots were binned into 2 separate bins, both classified as Photobacterium. All grey dots are unclassified contigs

wild adult salmon, the Mycoplasma level has been shown in 2 independent studies to be about $70 \%$ (Llewellyn et al. 2016), and above $90 \%$ (Holben et al. 2002). This suggests that the freshwater conditions, and potentially the lipid content of the feed, have an influence on the adult microbiota in Atlantic salmon.

Since tank and diet were not separated in our experimental setup, we were not able to separate their individual effects. Further experimentation is therefore needed to determine the exact contribution of the lipids in the feed. Independent of the cause, our experiments show that different freshwater conditions and/or diet can have a long-term effect on the seawater microbiota.

Although the body weight of the low LC-PUFA fish with high levels of gut Mycoplasma was about twothirds of the high LC-PUFA fish at sea entry, the difference between the groups evened out in seawater. This observation suggests no harmful or pathogenic effects of salmon gut Mycoplasma. Both the potential carbohydrate-utilizing lifestyle and the lower gene number may support a more benign lifestyle for salmon Mycoplasma than for other Mycoplasma (Hol- ben et al. 2002). There is also an increasing body of evidence for the dominance of Mycoplasma in the gut of other marine fishes (Bano et al. 2007, Song et al. 2016), possibly supporting a widespread mutualistic role of Mycoplasma in the marine environment. - Mycoplasma Although Photobacterium dominated in seawater for the high
LC-PUFA group, this genus showed Contig length a higher inter-individual variation - $25000 \quad 50000 \quad$ compared to Mycoplasma. Photo75000 100000 bacterium was also detected at the freshwater stage, unlike Mycoplasma. The gene content of the salmon Photobacterium indicated that this bacterium has evolved mechanisms to survive in oxidative environments with the genetic potential to produce catalase, oxidase and protective pigments. The presence of Photobacterium in other Arctic marine fishes (Song et al. 2016) may also suggest the general importance of this genus in the fish gut.

In a previous experiment, we found a major expansion of the bacterial load and increased alpha diversity after the transition to seawater (Rudi et al. 2018). This resembled our observation in the present experiment just before sea entry. It is therefore likely that fish in our previous experiment had not yet reached the adult-like microbiota upon seawater transition (Rudi et al. 2018). Furthermore, there may also be differences in seawater microbiota between controlled tank experiments, as compared to experiments at sea. A recent seawater tank experiment (Hartviksen et al. 2014) identified low levels of Mycoplasma in the gut content of fish with the same size and age as for the present experiment, while another recent study showed a high level of Mycoplasma in seawater (Dehler et al. 2017). These discrepancies highlight potential environmental and/or feed effects on the establishment of the gut microbiota (Rothschild et al. 2018). Unfortunately, however, no studies included the potential contribution of bacteria in the feed, or from the water in relation to the microbiota composition detected. These are aspects that should be included in future studies.

Taken together, we show a major succession and expansion of the gut microbiota of Atlantic salmon upon the transition from freshwater to the sea, with an establishment of a sea microbiota dominated by Mycoplasma-resembling that of wild salmon- 

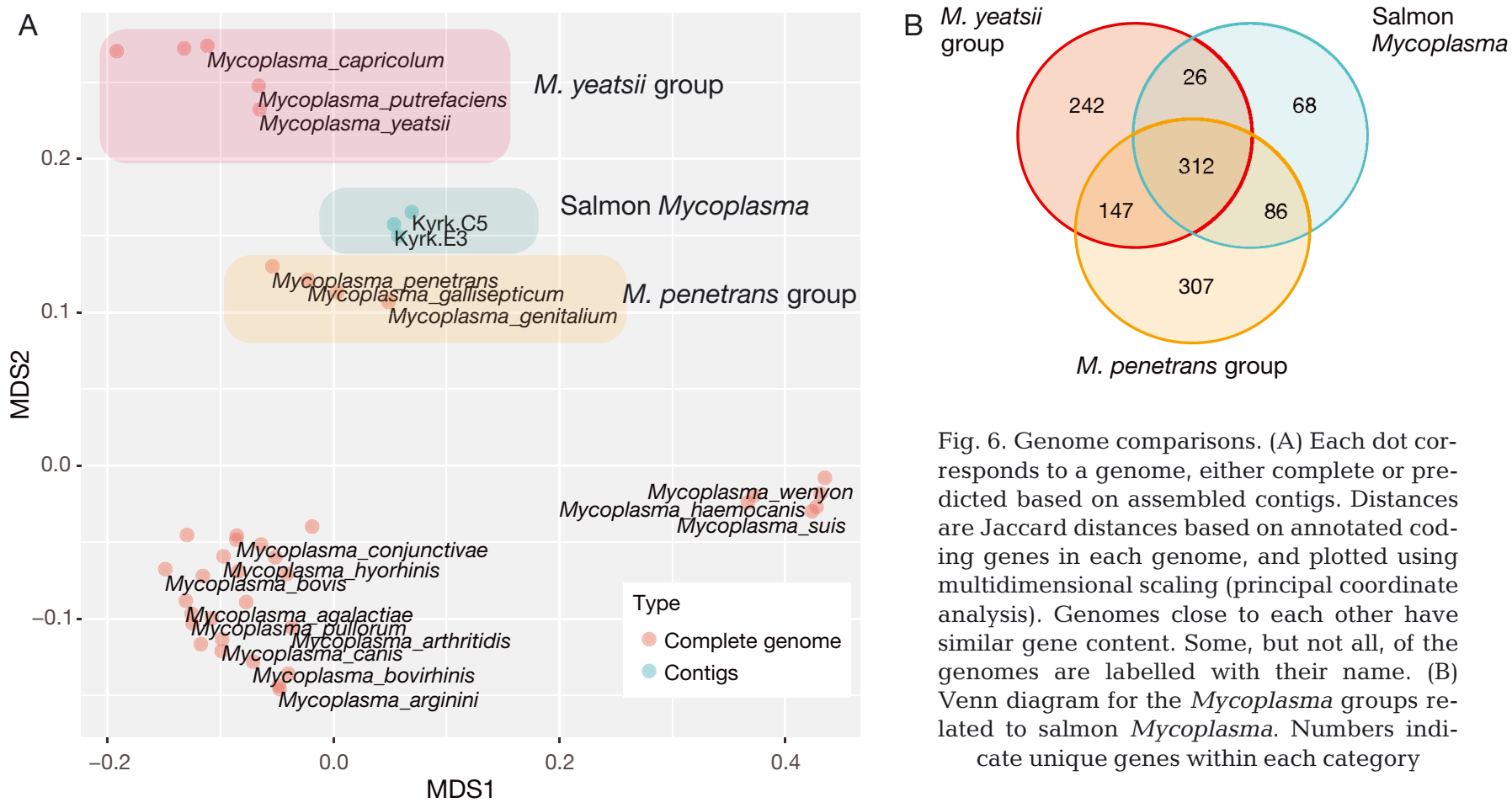

Fig. 6. Genome comparisons. (A) Each dot corresponds to a genome, either complete or predicted based on assembled contigs. Distances are Jaccard distances based on annotated coding genes in each genome, and plotted using multidimensional scaling (principal coordinate analysis). Genomes close to each other have similar gene content. Some, but not all, of the genomes are labelled with their name. (B) Venn diagram for the Mycoplasma groups related to salmon Mycoplasma. Numbers indicate unique genes within each category

depending on the initial gut microbiota composition in freshwater. Finally, metagenome sequencing supports no harmful effects of Mycoplasma, and this bacterium could play a pivotal role for the salmon hologenome (Limborg et al. 2018). Given the causal associations of Mycoplasma with salmon health, the possibility of intervention could have future consequences for salmon farming.

Data archive. Raw reads from the 16S rRNA gene and whole-genome shotgun sequencing are available in the NCBI sequence read archive (SRA) with accession numbers SRP132441 and SRP132676, respectively.

Acknowledgements. Cargill Innovation Center Dirdal is acknowledged for production of the fish feeds. Mari-Ann Østensen is acknowledged for helping with sampling of microbiota, Rolf Erik Olsen for giving helpful suggestions, and finally Nina Santi for the agreement of collaboration The work was financially supported by DigiSal NFR 248792 and GenoSysFat NFR 244164.

\section{LITERATURE CITED}

Angell IL, Hanssen JF, Rudi K (2017) Prokaryote species richness is positively correlated with eukaryote abundance in wastewater treatment biofilms. Lett Appl Microbiol 65:66-72

Bano N, DeRae Smith A, Bennett W, Vasquez L, Hollibaugh JT (2007) Dominance of Mycoplasma in the guts of the long-jawed mudsucker, Gillichthys mirabilis, from five California salt marshes. Environ Microbiol 9:2636-2641

Bell JG, Ghioni C, Sargent JR (1994) Fatty acid compositions of 10 freshwater invertebrates which are natural food organisms of Atlantic salmon parr (Salmo salar): a comparison with commercial diets. Aquaculture 128:301-313

Bolger AM, Lohse M, Usadel B (2014) Trimmomatic: a flexible trimmer for Illumina sequence data. Bioinformatics 30:2114-2120

Bråte J, Logares R, Berney C, Ree DK and others (2010) Freshwater Perkinsea and marine-freshwater colonizations revealed by pyrosequencing and phylogeny of environmental rDNA. ISME J 4:1144-1153

Buttigieg PL, Ramette A (2014) A guide to statistical analysis in microbial ecology: a community-focused, living review of multivariate data analyses. FEMS Microbiol Ecol 90:543-550

Caporaso JG, Kuczynski J, Stombaugh J, Bittinger K and others (2010) QIIME allows analysis of high-throughput community sequencing data. Nat Methods 7:335-336

Dehler CE, Secombes CJ, Martin SAM (2017) Seawater transfer alters the intestinal microbiota profiles of Atlantic salmon (Salmo salar L.). Sci Rep 7:13877

DeSantis TZ, Hugenholtz P, Larsen N, Rojas M and others (2006) Greengenes, a chimera-checked 16S rRNA gene database and workbench compatible with ARB. Appl Environ Microbiol 72:5069-5072

Edgar RC (2010) Search and clustering orders of magnitude faster than BLAST. Bioinformatics 26:2460-2461

Gillard G, Harvey TN, Gjuvsland A, Jin Y and others (2018) Diet and life stage associated remodeling of lipid metabolism regulation in the duplicated Atlantic salmon genome. Mol Ecol 27:1200-1213

Hartviksen M, Vecino JLG, Ringø E, Bakke AM and others (2014) Alternative dietary protein sources for Atlantic salmon (Salmo salar L.) effect on intestinal microbiota, intestinal and liver histology and growth. Aquacult Nutr 20:381-398

Holben WE, Williams P, Gilbert M, Saarinen M, Särkilahti 
LK, Apajalahti JH (2002) Phylogenetic analysis of intestinal microflora indicates a novel Mycoplasma phylotype in farmed and wild salmon. Microb Ecol 44:175-185

* Jin Y, Olsen RE, Gillard GB, Østensen MA and others (2018) A systemic study of lipid metabolism regulation in salmon fingerlings and early juveniles fed plant oil. Br J Nutr 120:653-664

Kang DD, Froula J, Egan R, Wang Z (2015) MetaBAT, an efficient tool for accurately reconstructing single genomes from complex microbial communities. PeerJ 3:e1165

Keegan KP, Glass EM, Meyer F (2016) MG-RAST, a metagenomics service for analysis of microbial community structure and function. Methods Mol Biol 1399:207-233

Langmead B, Salzberg SL (2012) Fast gapped-read alignment with Bowtie 2. Nat Methods 9:357-359

Leaver MJ, Bautista JM, Bjørnsson BT, Jønsson E and others (2008) Towards fish lipid nutrigenomics: current state and prospects for fin-fish aquaculture. Rev Fish Sci 16: 73-94

Lien S, Koop BF, Sandve SR, Miller JR and others (2016) The Atlantic salmon genome provides insights into rediploidization. Nature 533:200-205

Limborg MT, Alberdi A, Kodama M, Roggenbuck M, Kristiansen K, Gilbert MTP (2018) Applied hologenomics: feasibility and potential in aquaculture. Trends Biotechnol 36:252-264

LLlewellyn MS, McGinnity P, Dionne M, Letourneau J and others (2016) The biogeography of the Atlantic salmon (Salmo salar) gut microbiome. ISME J 10:1280-1284

Meyer F, Paarmann D, D'Souza M, Olson R and others

Editorial responsibility: Tim Dempster, Melbourne, Victoria, Australia
(2008) The metagenomics RAST server-a public resource for the automatic phylogenetic and functional analysis of metagenomes. BMC Bioinformatics 9:386

Nurk S, Meleshko D, Korobeynikov A, Pevzner PA (2017) metaSPAdes: a new versatile metagenomic assembler. Genome Res 27:824-834

Rodríguez JM, Murphy K, Stanton C, Ross RP and others (2015) The composition of the gut microbiota throughout life, with an emphasis on early life. Microb Ecol Health Dis 26:26050

Rothschild D, Weissbrod O, Barkan E, Kurilshikov A and others (2018) Environment dominates over host genetics in shaping human gut microbiota. Nature 555:210-215

Rudi K, Angell IL, Pope PB, Vik JO, Sandve SR, Snipen LG (2018) Stable core gut microbiota across the freshwaterto-saltwater transition for farmed Atlantic salmon. Appl Environ Microbiol 84:e01974-17

Seemann T (2014) Prokka: rapid prokaryotic genome annotation. Bioinformatics 30:2068-2069

Song W, Li L, Huang H, Jiang K and others (2016) The gut microbial community of Antarctic fish detected by $16 \mathrm{~S}$ rRNA gene sequence analysis. BioMed Res Int 2016: 3241529

Wood DE, Salzberg SL (2014) Kraken: ultrafast metagenomic sequence classification using exact alignments. Genome Biol 15:R46

Yu Y, Lee C, Kim J, Hwang S (2005) Group-specific primer and probe sets to detect methanogenic communities using quantitative real-time polymerase chain reaction. Biotechnol Bioeng 89:670-679

Submitted: April 3, 2018; Accepted: December 13, 2018 Proofs received from author(s): January 21, 2019 